\title{
Condições de Vida no Estado do Paraná: Análise Ecológica com Base em Variáveis do Censo Demográfico de 2000
}

\section{Living Conditions in the State of Paraná: Ecological Analysis Based on Variables of the 2000 Demographic Census}

\author{
Selma Maffei de Andrade1, Tiemi Matsuo ${ }^{2}$, Darli Antonio Soares ${ }^{3}$, \\ Regina Kazue Tanno de Souza ${ }^{4}$, Thais Aidar de Freitas Mathias ${ }^{4}$, \\ Maria Luiza Hiromi Iwakura ${ }^{5}$, Maria Angelina Zequim ${ }^{6}$
}

Resumo

\begin{abstract}
As condições de vida e as desigualdades sociais exercem poderosa influência nos níveis de saúde das populações. Neste sentido, este estudo, como parte de uma pesquisa que visa a analisar a relação entre condições de vida e mortalidade, apresenta uma proposta de categorização dos municípios do Paraná segundo condições de vida, com base em variáveis do Censo Demográfico de 2000, e analisa o poder discriminatório dos estratos definidos neste trabalho tendo por referência o Índice de Desenvolvimento Humano (IDH-M). Foram utilizadas dez variáveis do Censo Demográfico de 2000 e, após a análise de componentes principais e análise de cluster pelo método k-means, construídos cinco estratos (clusters) de municípios segundo condições de vida. Comparando os estratos, por meio de análise de variância (ANOVA) e teste de Tukey post-hoc, observaram-se desigualdades relativas à infra-estrutura urbana dos municípios (abastecimento de água e coleta de lixo) e de inserção social de seus residentes (renda e escolaridade). Na comparação com o IDH-M, observou-se que os clusters seguem um padrão semelhante, com classificação dos clusters em níveis mais altos de condições de vida à medida que houve aumento do IDH-M, com diferenças estatisticamente significativas, exceto para o componente "longevidade" do IDH-M. Os resultados indicam que os estratos construídos apresentaram poder discriminatório em relação às condições de vida, tendo como base as variáveis analisadas.
\end{abstract}

Palavras-chave: Condições de vida. Iniqüidade social. Desenvolvimento da comunidade.

\footnotetext{
1 Enfermeira, doutora em Saúde Pública, docente do Departamento de Saúde Coletiva da Universidade Estadual de Londrina. Email: semaffei@sercomtel.com.br

2 Bacharel em Matemática, doutora em Estatística, docente do Departamento de Matemática Aplicada da Universidade Estadual de Londrina.

3 Médico, doutor em Medicina, docente do Departamento de Saúde Coletiva da Universidade Estadual de Londrina.

4 Enfermeira, doutora em Saúde Pública, docente do Departamento de Enfermagem da Universidade Estadual de Maringá.

5 Odontóloga, mestre em Saúde Coletiva, coordenadora da Diretoria de Processamento e Análise de Dados em Saúde da Autarquia Municipal de Saúde de Londrina.

6 Geógrafa, mestranda em Geografia da Universidade Estadual de Londrina, Diretoria de Processamento e Análise de Dados em Saúde da Autarquia Municipal de Saúde de Londrina.
} 


\begin{abstract}
Living conditions and social inequalities exert a strong influence on the health status of populations. As part of a research that analyzes the relationship between living conditions and mortality rate, this study puts forward a proposal to classify municipalities in the State of Paraná, according to their living conditions. The classification was based on ten (10) variables of the 2000 Demographic Census, and on the analysis of the discriminatory power of the clusters developed by this study according to the municipality human development index (M-HDI). After having evaluated the municipalities main components and carried out a cluster analysis by the K-means method, five municipal clusters were developed, according to their living conditions. The comparative study of the clusters using the analysis of variance (ANOVA) and the post hoc Tukey test showed inequalities towards these municipalities urban infrastructure (water supply and garbage collection), and the social insertion of their population. Clusters followed a similar pattern when compared to the M-HDI. Those with increasing M-HDI were classified as having better living conditions, and with statistically significant differences, except for the "longevity" component. Results indicate that the clusters developed on the basis of the variables analyzed have a discriminatory power on their living conditions.
\end{abstract}

Key words: Living conditions. Social inequality. Community development.

\section{Introdução}

$\mathrm{Na}$ literatura especializada, observa-se, nos últimos anos, um crescente número de estudos que relacionam as condições de vida e as desigualdades sociais como determinantes do processo saúde/ doença. A investigação de trabalhos que tivessem como palavras-chave "fatores sócioeconômicos", "classe social", "renda" ou "pobreza", realizada na base de dados Medline, revelou um aumento de cerca de $58 \%$ em publicações a respeito dessa temática nos dez anos anteriores à pesquisa (KAPLAN; LYNCH, 1997). Esses diversos estudos têm demonstrado, consistentemente, forte associação entre más condições de vida e uma vasta gama de resultados de interesse à saúde, incluindo incidência de doenças, incapacidades, acesso a cuidados médicos e mortalidade prematura (BARNETT; ARMSTRONG; CASPER, 1997). A operacionalização do conceito de classe social, todavia, permanece sem proposta única, com diferentes indicadores e métodos utilizados na medição das condições de vida e das desigualdades sociais em diferentes locais e países (SOLLA, 1996).

No Brasil, a preocupação com a necessidade de aprofundamento das discussões teórico-metodológica e prática sobre o impacto das condições de vida e das desigualdades sociais no nível de saúde da população traduziu-se, de forma mais expressiva e organizada, na edição de dois livros pela Associação Brasileira de Pós-Graduação em Saúde Coletiva (ABRASCO): "Eqüidade e Saúde: contribuições da Epidemiologia" (BARATA et al., 1997) e "Condições de vida e situação de saúde" (BARATA, 1997). Nessas publicações, vários de seus autores denunciam as grandes iniqüidades sociais nas condições de vida presentes em nosso continente, com conseqüências perversas para a situação sanitária (CASTELLANOS, 1997). Além disso, os autores salientam que a investigação das relações entre saúde e sociedade é capaz de propiciar intervenções e mudanças sociais (BARROS, 1997), assim como enfatizam o papel da Epidemiologia nesse contexto. Segundo eles, a epidemiologia, ao revelar essas iniqüidades, contribui para a superação de proposições como a da focalização ou de atenção a "grupos de risco" e a implantação de políticas mais abrangentes, visando à melhoria da qualidade de vida e de saúde das populações (GOLDBAUM, 1997).

Qualquer que seja o delineamento utilizado, tendo como unidade de análise indivíduos ou agregados espaciais, estudos empíricos revelam, de forma consistente, os efeitos deletérios das más condições de vida e das desigualdades sociais na situação de saúde e no acesso a bens e serviços. No Brasil, 
Yazlle-Rocha, Simões e Guedes (1997), por exemplo, evidenciaram forte associação entre a ocupação da pessoa e a categoria de hospitalização (sistema público, convênio ou particular) em Ribeirão Preto, referindo que "à desigualdade diante dos riscos de agravos à saúde vem se acrescentar a decorrente das diferenças no sistema de financiamento médicohospitalar". Já em outro estudo, realizado em Salvador, as variáveis renda (capital econômico) e escolaridade (capital cultural) foram utilizadas para analisar os diferenciais de mortalidade nos diferentes espaços sociais da cidade, mostrando disparidades no risco de morrer, com excesso desse risco nos estratos com piores condições de vida (SILVA; PAIM; COSTA, 1999). Condições sociais desfavoráveis (medidas por categorização dos indivíduos em classes sociais) também foram determinantes no risco aumentado de vitimização por agressão física, para pessoas atendidas em prontosocorro de Sorocaba (GIANINI; LITVOC; ELUF NETO, 1999). Outro trabalho revelou maiores taxas de homicídios em espaços com concentração de favelas e de menor renda, no Rio de Janeiro, fato atribuível à deterioração nos padrões de coesão social, resultante das iniqüidades relativas dessas populações, comparadas à população em geral (SZWARCWALD et al., 1999).

Em face da importância do conhecimento da realidade de cada território em relação às condições de vida e de saúde, faz-se necessária a realização de estudos que apontem os espaços mais vulneráveis, no sentido de subsidiar políticas públicas que contribuam para reverter esse quadro. $\mathrm{O}$ presente artigo traz parte dos resultados de uma pesquisa que objetiva analisar a relação entre as condições de vida da população paranaense e sua mortalidade. Serão apresentados aqui dados referentes à categorização dos municípios em estratos segundo condições de vida, por intermédio da análise de algumas variáveis marcadoras de condições de vida da população e de infraestrutura desses municípios, derivadas do Censo Demográfico de 2000 (IBGE, 2003). Busca-se identificar áreas com condições menos favoráveis à vida humana e compará-las ao Índice de Desenvolvimento Humano Municipal (IDH-M) de 2000, divulgado recentemente pelo Instituto Paranaense de Desenvolvimento Econômico e Social (2003).

Faz-se necessário esclarecer que este índice (o IDH-M) não pôde ser utilizado na presente pesquisa como variável independente em relação à variável dependente mortalidade, já que incorpora, entre seus componentes, a esperança de vida ao nascer (longevidade da população), índice baseado em dados de mortalidade. Dessa forma, a análise da relação entre condições de vida e mortalidade, utilizando o IDH-M, provocaria, possivelmente, correlações espúrias.

\section{Metodologia}

Foi realizado um estudo de agregados (ecológico), tendo como unidades de análise os 399 municípios do estado do Paraná, região Sul do Brasil, que contava, em 2000, com uma população de 9.563 .458 habitantes (IBGE, 2003).

A classificação desses municípios, segundo estratos de condições de vida, baseou-se na análise de dez variáveis derivadas do censo demográfico de 2000 (IBGE, 2003). A seleção dessas variáveis, a seguir descritas, buscou contemplar aspectos relativos à infra-estrutura dos municípios e de inserção social das famílias:

a) proporção de domicílios particulares permanentes cujos responsáveis não têm rendimento ou que recebem até 2 salários mínimos (RENDA < 2 S.M.);

b) rendimento médio nominal mensal dos responsáveis por domicílios particulares permanentes, entre aqueles com rendimento (RENDA1);

c) rendimento mediano nominal mensal dos responsáveis por domicílios particulares permanentes, entre aqueles com rendimento (RENDA2); 
d) proporção de domicílios particulares permanentes sem banheiro ou sanitário (SEMBANH);

e) proporção de domicílios particulares permanentes com coleta de lixo (LIXO);

f) proporção de domicílios particulares permanentes com abastecimento de água da rede geral (AGUA);

g) proporção de responsáveis por domicílios particulares permanentes sem instrução ou com menos de 8 anos de estudo (ESTUDO7);

h) proporção da população residente vivendo em domicílios improvisados (DOMIMP);

i) proporção da população com 10 anos ou mais de idade alfabetizada (ALFAB10);

j) taxa de urbanização (URBANIZAÇÃO).

Utilizando a técnica de análise de componentes principais (ACP), por meio do programa estatístico SAS (SAS, 1989), foram selecionadas as variáveis correlacionadas com os componentes que melhor explicavam a variância total. O primeiro componente (AGUA, LIXO e URBANIZAÇÃO) e o segundo (RENDA < 2 S.M. e ALFAB10), ambos com autovalores $>1$, valor este que tem sido utilizado na literatura (GUIMARÃES et al., 2003), explicavam $76,6 \%$ da variação entre os municípios, sendo as variáveis desses dois componentes, portanto, utilizadas na análise.

A seguir, os municípios foram ordenados da pior para a melhor situação, para cada uma dessas variáveis, recebendo pontos correspondentes ao posto obtido, sendo realizado, então, o somatório da pontuação dessas cinco variáveis, o que resultou em um determinado escore. Teoricamente, o município com piores condições receberia um total de cinco pontos (um ponto referente ao primeiro posto vezes as cinco variáveis) e o melhor, 1995 pontos (todas as cinco variáveis no $399^{\circ}$ posto). Na prática, a pontuação variou de 58 a 1993.

Utilizando o programa SAS (SAS, 1989) foi realizada, então, a análise de cluster pelo método não-hierárquico $k$-means, tendo como base os escores obtidos, com o objetivo de agregar municípios em estratos (clusters) com a máxima homogeneidade interna e a máxima heterogeneidade entre os estratos (CARVALHO; CRUZ; NOBRE, 1997). Esse segundo tipo de análise resultou em cinco estratos, com os municípios classificados da pior (cluster 1) para a melhor situação (cluster 5).

Posteriormente, foi feita comparação do Índice de Desenvolvimento Humano (IDH-M) médio e de seus componentes (renda - IDH-R, educação - IDHE e longevidade - IDH-L) dos municípios em cada um dos cinco estratos de condições de vida, a fim de verificar o poder discriminatório dos estratos em relação às condições de vida. A análise de variância (ANOVA) e o teste de Tukey post-hoc foram utilizados nessa comparação (WAYNE, 1995).

\section{Resultados e discussão}

Dos 399 municípios paranaenses, 48 (12,0\%) foram classificados no estrato de piores condições de vida das famílias e de estrutura urbana (cluster 1; 91 (22,8\%) foram classificados no cluster 2; 96 $(24,1 \%)$, no cluster $3 ; 90(22,6 \%)$ no cluster 4 ; e 74 $(18,5 \%)$ no cluster 5 , sendo este o de melhores condições. As tabelas 1 e 2 apresentam as principais estatísticas descritivas para esses estratos. Nota-se, por esses resultados, que as desigualdades relativas à infra-estrutura de coleta de lixo e de abastecimento de água dos municípios, além da renda do responsável pela família, são grandes, especialmente se o cluster 1 (piores condições de vida) for comparado com o cluster 5 (de melhores condições). Por exemplo, enquanto no cluster 1 a proporção média de domicílios com coleta regular de lixo é de $31,45 \%$ (variando de $8,75 \%$ a $57,75 \%$ ), no cluster 5 essa média é de $89,24 \%$ dos domicílios (com variação de $63,31 \%$ a $99,60 \%)$.

Comparando os clusters segundo cada variável isoladamente, por intermédio do teste de Tukey, observou-se que estes diferem entre si de forma estatisticamente significativa, exceto para a variável 
taxa de alfabetização entre pessoas com 10 anos ou mais (ALFAB10). Para esta, houve diferença apenas entre os clusters 1 e 5. Os clusters 2, 3 e 4, intermediários, não apresentaram diferença entre si, distinguindo-se apenas em relação ao cluster de pior condição de vida (1) e ao de melhor situação (5). Este resultado, possivelmente, é decorrente da relativa homogeneidade da taxa de alfabetização no Estado, o que reduz sua capacidade de evidenciar diferenciais nas condições de vida e aponta para a necessidade de outros parâmetros para avaliação desse componente da qualidade de vida, especialmente nos clusters intermediários, por serem mais homogêneos em relação a esta variável.

Tabela 1. Estatísticas descritivas para proporção de domicílios com coleta de lixo, ligados à rede de abastecimento de água e taxa de urbanização, segundo clusters de condições de vida, Paraná, 2000.

\begin{tabular}{|c|c|c|c|c|c|}
\hline \multirow{2}{*}{ VARIÁVEL } & \multicolumn{5}{|c|}{ Estatísticas descritivas } \\
\hline & Média & Desvio padrão & Mediana & Mínimo & Máximo \\
\hline \multicolumn{6}{|l|}{ LIXO } \\
\hline Cluster 1 & 31,45 & 13,51 & 32,54 & 8,75 & 57,75 \\
\hline Cluster 2 & 48,86 & 13,12 & 50,42 & 15,71 & 75,18 \\
\hline Cluster 3 & 64,90 & 9,72 & 66,23 & 37,26 & 80,52 \\
\hline Cluster 4 & 77,12 & 7,83 & 78,02 & 53,62 & 95,27 \\
\hline Cluster 5 & 89,24 & 6,39 & 89,40 & 63,31 & 99,60 \\
\hline \multicolumn{6}{|l|}{ ÁGUA } \\
\hline Cluster 1 & 38,40 & 14,13 & 40,10 & 13,64 & 66,82 \\
\hline Cluster 2 & 56,12 & 14,21 & 59,04 & 19,67 & 83,77 \\
\hline Cluster 3 & 69,07 & 9,77 & 70,82 & 49,36 & 86,72 \\
\hline Cluster 4 & 79,85 & 6,93 & 80,14 & 57,63 & 94,26 \\
\hline Cluster 5 & 89,19 & 4,64 & 89,04 & 78,07 & 98,61 \\
\hline \multicolumn{6}{|c|}{ URBANIZAÇÃO } \\
\hline Cluster 1 & 28,79 & 11,77 & 28,94 & 8,26 & 50,17 \\
\hline Cluster 2 & 45,71 & 13,89 & 48,58 & 9,80 & 72,21 \\
\hline Cluster 3 & 62,54 & 11,12 & 63,02 & 29,42 & 81,91 \\
\hline Cluster 4 & 72,88 & 12,44 & 75,94 & 12,25 & 92,21 \\
\hline Cluster 5 & 88,39 & 7,52 & 89,59 & 59,83 & 100,00 \\
\hline
\end{tabular}

Tabela 2. Estatísticas descritivas para proporção de responsáveis pelos domicílio com renda $<2$ salários mínimos e taxa de alfabetização entre pessoas com 10 anos ou mais de idade segundo clusters de condições de vida, Paraná, 2000.

\begin{tabular}{|c|c|c|c|c|c|}
\hline \multirow{2}{*}{ VARIÁVEL } & \multicolumn{5}{|c|}{ Estatísticas descritivas } \\
\hline & Média & Desvio padrão & Mediana & Mínimo & Máximo \\
\hline RENDA < 2 S.M. & & & $\bullet$ & & \\
\hline Cluster 1 & 78,45 & 3,20 & 78,68 & 71,89 & 87,16 \\
\hline Cluster 2 & 71,63 & 5,32 & 72,34 & 55,51 & 82,52 \\
\hline Cluster 3 & 67,17 & 6,43 & 68,18 & 33,31 & 80,44 \\
\hline Cluster 4 & 61,38 & 6,80 & 61,84 & 35,32 & 74,12 \\
\hline Cluster 5 & 49,27 & 7,86 & 49,30 & 25,24 & 62,88 \\
\hline \multicolumn{6}{|l|}{ ALFAB10 } \\
\hline Cluster 1 & 82,03 & 3,90 & 81,62 & 74,32 & 91,67 \\
\hline Cluster 2 & 85,11 & 3,74 & 84,80 & 79,30 & 94,15 \\
\hline Cluster 3 & 86,49 & 3,74 & 85,87 & 79,41 & 94,83 \\
\hline Cluster 4 & 87,81 & 3,27 & 87,20 & 81,25 & 97,72 \\
\hline Cluster 5 & 91,32 & 2,69 & 91,15 & 85,65 & 96,86 \\
\hline
\end{tabular}

A figura 1 apresenta a localização geográfica dos municípios paranaenses de acordo com a classificação dos clusters. Observa-se concentração de municípios classificados no estrato de piores condições de vida (cluster 1) na região central do estado, além de alguns localizados na divisa com o estado de São Paulo, no Vale da Ribeira, e alguns outros dispersos na Região Sul do Estado. Esse mapa aponta uma situação semelhante à identificada pelo Índice de Desenvolvimento Humano Municipal do estado, elaborado pelo Instituto Paranaense de Desenvolvimento Econômico e Social (2003), índice que leva em conta indicadores de educação (alfabetização e frequiência escolar), longevidade e renda per capita da população. 


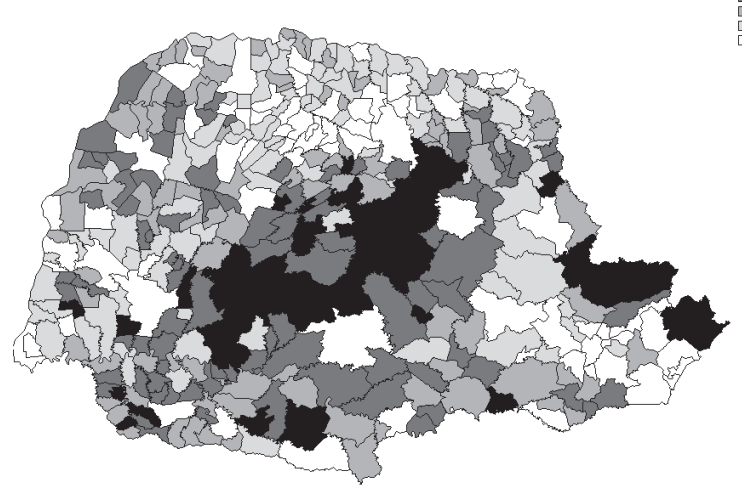

Figura 1. Distribuição geográfica dos municípios paranaenses segundo classificação por clusters de condições de vida, 2000.

A identificação de áreas menos favoráveis à vida humana é fundamental para orientar estratégias que visem ao aumento da eqüidade na qualidade de vida e de saúde, buscando reduzir ou eliminar desigualdades consideradas injustas (SILVA; BARROS, 2002). Para Castellanos (1997), a busca de eqüidade, usando como exemplo as taxas de mortalidade na região das Américas, remete à necessidade de se comparar essas taxas com o que de melhor já foi atingido por um grupo social ou país, sendo este um valor de referência (meta). A diferença entre os valores das taxas de cada grupo social, região ou país e esse valor de referência é o que esse autor denomina de "brechas redutíveis de mortalidade" (BRM). O mesmo raciocínio a respeito das BRM poderia ser aplicado a outros aspectos da vida humana, como, por exemplo, em relação às diferenças entre grupos, municípios ou regiões em termos de serviços e bens sociais que deveriam ser universais (água tratada, esgotamento sanitário, assistência à saúde, escolaridade, entre outros), que poderiam ser denominadas de "brechas redutíveis de falta de acesso" a esses serviços.

Na comparação entre os clusters construídos para este estudo e o IDH-M, observou-se uma relação diretamente proporcional, ou seja, os clusters com piores condições de vida também apresentaram piores médias do IDH-M (Figura 2) e essa diferença foi estatisticamente significante $(\mathrm{p}<0,001$; ANOVA e teste de Tukey).



Figura 2. Índice de desenvolvimento humano municipal (IDH-M) médio (e respectivo intervalo de confiança de 95\%) de acordo com os clusters de condições de vida no Paraná, 2000.

Com relação aos componentes do IDH-M, isto é, quanto aos indicadores de renda da população (IDH$\mathrm{R}$ ), de educação (alfabetização e frequiência escolar) (IDH-E) e de longevidade (IDH-L), verificaram-se diferenças estatisticamente significativas entre todos os clusters para o IDH-E e IDH-R, também de forma diretamente proporcional (melhoria das condições de vida dos clusters significando aumento da média do componente do IDH). Apesar de apresentar um índice médio crescente do cluster 1 para o 5, o indicador de longevidade (IDH-L) revelou um padrão diferente, pois, como mostra a figura 3 , houve sobreposição dos intervalos de confiança de 95\% entre os clusters consecutivos, ou seja, entre o cluster 1 e 2 , entre o 2 e 3 , entre o 3 e 4 e entre o 4 e 5. Portanto, diferiram, de forma estatisticamente significativa, o cluster 1 em relação ao 3, 4 e 5; o cluster 2 em relação ao 4 e 5; o cluster 3 em relação ao 1 e 5 ; o cluster 4 em relação ao 2 e 1 e, finalmente, o cluster 5 em relação aos clusters 1,2 e 3 . 


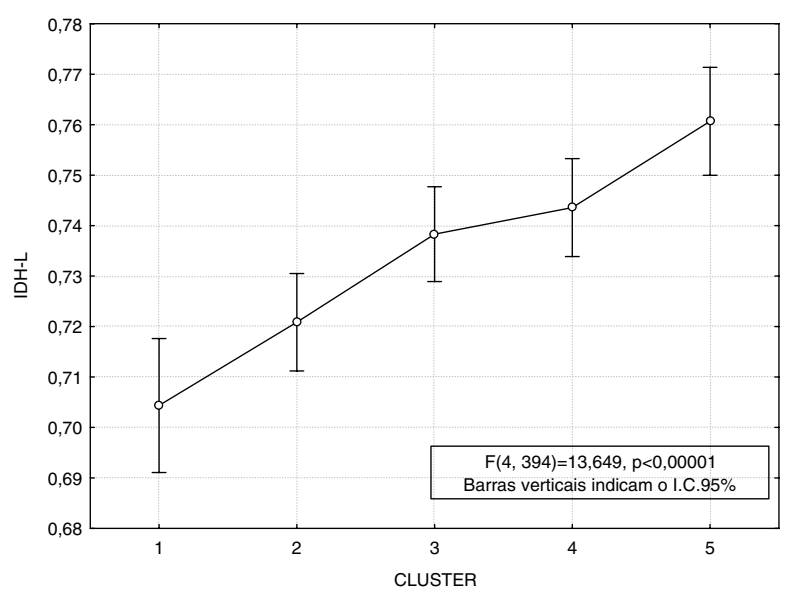

Figura 3. IDH-L (longevidade) médio (e respectivos intervalos de confiança de 95\%) de acordo com cada cluster de condições de vida no Paraná, 2000.

Esses resultados indicam que os clusters construídos para este estudo, que visa, numa próxima etapa, analisar a relação entre condições de vida e mortalidade no estado do Paraná. Eles discriminam adequadamente os municípios, pois, exceto pela variável taxa de alfabetização entre pessoas com 10 anos ou mais, todos os clusters diferiram significativamente entre si, com um gradiente de melhora nas taxas das variáveis analisadas do cluster de pior para o de melhor condição de vida. $\mathrm{Na}$ comparação com o Índice de Desenvolvimento Humano Municipal, observou-se, também, elevação significativa da média desse índice nos clusters, exceto para o componente relativo à "longevidade", o que pode indicar que agravos que não estejam altamente relacionados às condições de vida possam estar ceifando vidas de forma prematura nos municípios em que as condições são mais favoráveis. Contudo, mesmo para este componente, observouse uma tendência de aumento médio do índice do estrato de pior condição de vida para o de melhor.

A respeito das desigualdades em saúde, Starfield (2002) afirma que essas não ocorrem de forma aleatória. Para essa autora, a saúde dos indivíduos e das comunidades é influenciada por uma gama de fatores (sociais, políticos, ambientais, genéticos, entre outros) que se inter-relacionam em uma rede complexa, tornando-se um desafio extraordinário entender a gênese das iniqüidades em saúde. Buscar conhecer e revelar as relações entre as condições de vida e a saúde de indivíduos e comunidades é, dessa forma, essencial para as transformações necessárias (ROJAS, 2000).

O presente trabalho buscou, portanto, com base em dados secundários, amplamente acessíveis, propor um indicador composto de condições de vida capaz de revelar áreas do estado do Paraná com condições menos propícias à vida. Com a metodologia proposta foi possível identificar essas áreas no Estado e revelar um padrão semelhante, embora não idêntico, ao descrito pelo Índice de Desenvolvimento Humano Municipal (INSTITUTO PARANAENSE DE DESENVOLVIMENTO ECONÔMICO E SOCIAL, 2003), com regiões mais carentes concentradas em determinadas regiões do Estado. Nesse contexto, cabe à sociedade e seus governantes discutir e buscar viabilizar políticas e estratégias que visem a combater essas desigualdades e a promover uma melhor qualidade de vida e de saúde a todos os cidadãos paranaenses.

$\mathrm{Na}$ continuidade deste estudo, tendo como hipótese de que as condições concretas de vida exercem influência no perfil de mortalidade dos municípios, pretende-se analisar os diferenciais no risco de morrer segundo os distintos estratos de condições de vida no estado do Paraná.

\section{Agradecimentos}

À Fundação Araucária e ao Conselho Nacional de Desenvolvimento Científico e Tecnológico (CNPq), pelo apoio financeiro (Processo 521030/994 - Plano Sul de Pesquisa e Pós-Graduação). 


\section{Referências}

BARATA, R. B (Org.). Condições de vida e saúde. Rio de Janeiro: ABRASCO, 1997.

BARATA, R. B.; BARRETO, M. L.; ALMEIDA, F. N.; VERAS, N. de; PEIXOTO, R. (Org.). Eqüidade e saúde: contribuições da Epidemiologia. Rio de Janeiro: ABRASCO, 1997.

BARNETT, E.; ARMSTRONG, D. L.; CASPER, M. L. Social class and premature mortality among men: a method for state-based surveillance. American Journal of Public Health, Washington, v.87, n.9, p.1521-1525, set. 1997.

BARROS, M. B. A. Epidemiologia e superação das iniqüidades em saúde. In: BARATA, R. B.; BARRETO, M. L.; ALMEIDA, F. N.; VERAS, N de; PEIXOTO, R. (Org.). Eqüidade e saúde: contribuições da Epidemiologia. Rio de Janeiro: ABRASCO, 1997. p.161-76.

CARVALHO, M. S.; CRUZ, O. G.; NOBRE, F. F. Perfil de risco: método multivariado de classificação sócio-econômica de microáreas urbanas: os setores censitários da região metropolitana do Rio de Janeiro. Cadernos de Saúde Pública, Rio de Janeiro, v.13, n.4, p.635-345, dez. 1997.

CASTELLANOS, P. L. Epidemiologia, saúde pública, situação de saúde e condições de vida. Considerações conceituais. In: BARATA, R. B. (Org.). Condições de vida e saúde. Rio de Janeiro: ABRASCO, 1997. p.31-75.

GIANINI, R. J.; LITVOC, J.; ELUF NETO, J. Agressão física e classe social. Revista de Saúde Pública, São Paulo, v.33, n.2, p.180-186, abr. 1999.

GOLDBAUM, M. A epidemiologia em busca da eqüidade em saúde. In: BARATA, R. B.; BARRETO, M. L.; ALMEIDA, F. N.; VERAS, N de; PEIXOTO, R. (Org.). Eqüidade e saúde: contribuições da Epidemiologia. Rio de Janeiro: ABRASCO; 1997. p.63-80.

GUIMARÃES, M. J. B.; MARQUES, N. M.; MELO FILHO, D. A.; SZWARCWALD, C. L. Condição de vida e mortalidade infantil: diferenciais intra-urbanos no Recife, Pernambuco, Brasil. Cadernos de Saúde Pública, Rio de Janeiro, v.19, n.5, p.1413-1424, out. 2003.

IBGE - (Instituto Brasileiro de Geografia e Estatística). População residente, Paraná. Disponível em <http:// www.ibge.gov.br>. Acesso em: 2 fev. 2003.

INSTITUTO PARANAENSE DE DESENVOLVIMENTO ECONÔMICO E SOCIAL - IPARDES. Índice de Desenvolvimento Humano Municipal - IDH-M 2000: anotações sobre o desempenho do Paraná. Curitiba: Instituto Paranaense de Desenvolvimento Econômico e Social, 2003. Disponível em <http://www.pr.gov.br/ipardes/ pdf/idmh_2000.pdf>. Acesso em: 5 ago. 2003.

KAPLAN, G. A.; LYNCH, J. W. Editorial: whiter studies on the socioeconomic foundations of population health [Editorials and annotations]. American Journal of Public Health, Washington, v.87, n.9, p.1409-1411, set. 1997.

ROJAS, L. I. Desigualdades espaciales del bienestar y la salud en América Latina. Problemas éticos y metodológicos. In: BRICEÑO-LEÓN; MINAYO, M. C. S.; COIMBRA JR., C. E. A. (Coord.). Salud y eqüidad: una mirada desde las ciencias sociales. Rio de Janeiro: Editora FIOCRUZ, 2000. p.121-135.

SAS. Institute Inc. SAS/STAT User's Guide. Version 6. 14.ed. Cary: SAS Institute Inc., 1989.

SILVA, L. M. V.; PAIM, J. S.; COSTA, M. C. N. Desigualdades sociais na mortalidade, espaço e estratos sociais. Revista de Saúde Pública, São Paulo, v.33, n.2, p.187-197, abr. 1999.

SILVA, J. B.; BARROS, M. B. A. Epidemiologia e desigualdade: notas sobre a teoria e a história. Revista Panamericana de Salud Pública, Washington, v.12, n.6, p.375-383, dez. 2002.

SOLLA, J. J. S. P. Problemas e limites da utilização do conceito de classe social em investigações epidemiológicas: uma revisão crítica da literatura. Cadernos de Saúde Pública, Rio de Janeiro, v.12, n.1, p.207-216, abr. 1996.

STARFIELD, B. Equity and health: a perspective on nonrandom distribution of health in the population. Revista Panamericana de Salud Pública, Washington, v.12, n.6, p.384-387, dez. 2002.

SZWARCWALD, C. L.; BASTOS, F. I.; VIACAVA, F.; ANDRADE, C. L. T. Income inequality and homicide rates in Rio de Janeiro, Brazil. American Journal of Public Health, Washington, v.89, n.6, p.845-850, jun. 1999.

WAYNE, D. W. Biostatistics: a foundation for analysis in the health sciences. 6.ed. New York: John Wiley, 1995. p.295-298.

YAZLLE-ROCHA, J. S.; SIMÕES, B. J. G.; GUEDES, G. L. M. Assistência hospitalar como indicador de desigualdades sociais. Revista de Saúde Pública, São Paulo, v.31, n.5, p.479-487, out. 1997. 